\title{
DONOR HEART PRESERVATION WITH A NOVEL HYPERPOLARIZING SOLUTION: SUPERIOR PROTECTION COMPARED WITH UNIVERSITY OF WISCONSIN SOLUTION
}

Eric M. Hoenicke, MD

Xiwu Sun, MD, PhD

Robert G. Strange, Jr, MD

Ralph J. Damiano, Jr, MD
Objectives: A donor heart preservation solution was designed to use hyperpolarized arrest with the adenosine triphosphate-sensitive potassium-channel opener pinacidil. This solution contained concentrations of potassium, sodium, calcium, magnesium, lactobionate, and the buffer histidine specifically chosen to minimize intracellular calcium accumulation associated with prolonged ischemia.

Methods: Twenty-four rabbit hearts were randomly assigned to receive 1 of 3 preservation solutions in a crystalloid-perfused Langendorff model: (1) prototype solution containing a $0.5 \mathrm{mmol} / \mathrm{L}$ concentration of pinacidil, (2) prototype solution without pinacidil as control, and (3) University of Wisconsin solution. Thirty minutes of initial perfusion preceded baseline data acquisition. Data comprised left ventricle pressure-volume curves generated by inflating an intraventricular latex balloon. After cardioplegic administration, hearts underwent 4 hours of hypothermic storage, followed by 60 minutes of reperfusion and postischemic data acquisition.

Results: Postischemic developed pressure was better preserved by pinacidil solution $(92.4 \% \pm 4.5 \%)$ than by the control $(74.9 \% \pm 3.4 \%, P=.01)$ and University of Wisconsin solutions $(66.7 \% \pm 5.1 \%, P=.001)$. Diastolic negative $\mathrm{dP} / \mathrm{dT}$ was better preserved by pinacidil solution $(104.4 \% \pm 10.2 \%)$ than by the control $(80.2 \% \pm 4.2 \%, P=.034)$ and University of Wisconsin solutions $(71.7 \% \pm 7.0 \%, P=.015)$. Diastolic compliance, expressed as baseline/postischemic diastolic slope ratios, was more poorly preserved by University of Wisconsin solution $(0.67 \pm 0.07)$ than by the pinacidil $(0.88 \pm$ $0.05, P=.041)$ and control solutions $(0.87 \pm 0.05, P=.021)$. Postischemic coronary flow was higher in hearts exposed to pinacidil solution $(77.8 \% \pm$ $3.0 \%)$ than in those exposed to the control $(66.8 \% \pm 2.4 \%)$ and University of Wisconsin solutions $(70.9 \% \pm 4.0 \%, P=.07)$.

Conclusions: The superiority of the pinacidil solution in this experiment demonstrated that hyperpolarized arrest with potassium-channel openers improves donor heart preservation when administered in a novel histidinebuffered lactobionate-enriched vehicle. (J Thorac Cardiovasc Surg 2000;120:746-54)
0 ne of the most significant limitations in heart transplantation is the relatively short preservation time that current preservation solutions offer. Safe organ preservation can be maintained for only 4 hours.

From the Department of Surgery, Division of Cardiothoracic Surgery, The Milton S. Hershey Medical Center, Pennsylvania State University, Hershey, Pa.

Supported by National Institutes of Health grants HL-51032 (R.J.D.) and Penn State College of Medicine Department of Surgery Feasibility Grant (E.M.H., R.J.D.)

Received for publication Feb 22, 2000; revisions requested April 17, 2000; revisions received May 31, 2000; accepted for publication June 14, 2000.
Extending donor heart preservation time would offer several advantages. Surgical results would likely improve because it has been demonstrated with present solutions that cold ischemic times inversely correlate with postoperative survival. ${ }^{1}$ It also would allow for

Address for reprints: Ralph J. Damiano, Jr, MD, Department of Surgery, Division of Cardiac Surgery, Washington University School of Medicine, Campus Box 8234, Suite 3108 Queeny Tower, One Barnes-Jewish Hospital Plaza, St Louis, MO 63110 (E-mail: damianor@msnotes.wustl.edu).

Copyright (C) 2000 by The American Association for Thoracic Surgery

0022-5223/2000\$12.00+ $0 \quad \mathbf{1 2 / 1 / 1 0 9 7 0 5}$

doi:10.1067/mtc.2000.109705 
completion of HLA tissue typing, which currently takes approximately 8 hours. This is important because long-term survival after heart transplantation is associated with HLA compatibility. ${ }^{2}$ Finally, extension of the tolerable cold ischemic time would also expand the procurement area for each heart transplant institution and potentially change the status of the operation from emergency to urgent.

All current strategies of long-term preservation for heart transplantation involve the use of hypothermic, hyperkalemic, cardioplegic solutions. These solutions rapidly induce depolarized arrest of the donor heart by means of a rapid increase in extracellular potassium. However, depolarized arrest is associated with ionic and metabolic imbalances that have been shown to lead to arrhythmias, abnormal regulation of intracellular second messengers, activation of adenosine triphosphate (ATP)-wasting cytosolic and membrane-bound enzyme systems, ischemia-reperfusion injury, and myocyte swelling. ${ }^{3}$ One of the pathophysiologic processes considered to be responsible for these sequelae has been intracellular calcium accumulation. ${ }^{4}$

Depolarized arrest leads to intracellular calcium accumulation by 3 distinct but interrelated mechanisms. ${ }^{4}$ As the membrane becomes depolarized, voltage-sensitive calcium channels open, activating an inward calcium window current. Intracellular sodium concentration also increases as the sodium window current is activated. This increased cytosolic sodium concentration leads to intracellular calcium accumulation through activation of the sodium-calcium exchanger. The third mechanism results from cytosolic calcium-induced calcium release from the sarcoplasmic reticulum.

Our laboratory has proposed that adopting an alternative strategy of hyperpolarized arrest may alleviate many of these untoward sequelae. ${ }^{5-7}$ To hyperpolarize the cell membrane, we have administered drugs that open ATP-sensitive potassium channels. These potassium-channel openers have been shown to be superior or equivalent to depolarized arrest during both short and long periods of global ischemia. ${ }^{5-7}$ Other laboratories have corroborated our findings that hyperpolarized arrest may be superior to depolarized arrest for myocardial protection. ${ }^{8,9}$ Recently, we have shown that ATP-sensitive potassium-channel openers prevent intracellular calcium accumulation during global ischemia. $^{10}$

To optimize our hyperpolarizing preservation solution, we formulated the composition to minimize intracellular calcium overload, prevent myocyte swelling, and promote anaerobic glycolytic flux. To avoid hyperkalemic depolarization, we designed the solution with a physiologic potassium concentration of $5 \mathrm{mmol} / \mathrm{L}$. To reduce sodium influx, thereby reducing calcium entry through the sodium-calcium exchanger, we formulated the solution with a low (intracellular) sodium concentration and augmented it with a sodium channel blocker, procaine. Elevated magnesium and micromolar calcium concentrations were included because this ratio of high magnesium and low calcium has also been associated with decreased intracellular calcium. ${ }^{11}$ The solution was enriched with lactobionate to chelate extracellular calcium. The alkaline essential amino acid histidine was included in high concentrations to buffer intracellular protons because of its favorable $\mathrm{pKa}(6.8$ at $25^{\circ} \mathrm{C}$ ). Theoretically, histidine was added to prevent intracellular sodium increase through the sodium-proton exchanger, subsequently reducing inward calcium flux by the sodium-calcium exchanger. Previous work has also implicated cellular swelling as an etiologic factor in postcardioplegia myocardial stunning. ${ }^{12}$ Therefore, cardioprotective features that limit cellular edema were built into the solution, including the osmotic impermeants hydroxyethyl starch and mannitol and a low concentration of chloride. ${ }^{13,14}$ Histidine was also included to stimulate glycolytic flux, ${ }^{15}$ with high concentrations of glucose as a metabolic substrate and insulin incorporated into the solution.

This study compared the cardioprotective effects of the potassium-channel opener pinacidil administered in our prototype preservation solution and the preservation solution without pinacidil (control) with the extensively used University of Wisconsin (UW) solution during prolonged ischemia.

\section{Methods}

Adult New Zealand White rabbits of either sex weighing 2.8 to $4.2 \mathrm{~kg}$ were used in this study. All animals received humane care in American Association for Accreditation of Laboratory Animal Care International-accredited (\#00036), US Department of Agriculture-registered (\#52-R-007) facilities in compliance with the "Principles of Laboratory Animal Care" formulated by the National Society for Medical Research and the "Guide for the Care and Use of Laboratory Animals" prepared by the Institute of Laboratory Animal Resources and published by the National Institutes of Health (National Institutes of Health publication No. 85-23, revised 1985).

Animal preparation. Anesthesia was accomplished by an intramuscular injection of acepromazine $(1.0 \mathrm{mg} / \mathrm{kg})$ and xylazine $(17.5 \mathrm{mg} / \mathrm{kg})$, followed by ketamine $(62.5 \mathrm{mg} / \mathrm{kg})$. A tracheotomy was performed, and mechanical ventilation (model 683; Harvard Apparatus, S. Natick, Mass) was implemented through an endotracheal tube with $100 \%$ oxygen. Intravenous heparin was administered (2500 U) through an ear vein. After heparinization, a sternotomy and rapid cardiectomy were performed, and the heart was immediately placed into a bowl of $4{ }^{\circ} \mathrm{C}$ electrolyte solution (Plasma-Lyte; Baxter Healthcare Corp, Deerfield, Ill). The aorta was sewn 
to a cannula and rapidly attached to a crystalloid perfusion column. A polyethylene vent was inserted through the apex of the left ventricle.

A fluid-filled latex balloon was inserted into the left ventricle and secured to the mitral valve anulus with a purse-string suture (5-0 Prolene; Ethicon, Inc, Somerville, NJ). The intraventricular balloon was connected with polyethylene tubing to a pressure transducer (model P23ID; Gould Instrument Systems, Inc, Valley View, Ohio) and amplifier (model 204615-50; Gould). Two needle electrodes were placed on the left ventricular (LV) epicardium to monitor the bipolar ventricular electrogram. The signal was filtered between 0.05 and 1000 $\mathrm{Hz}$. Two other electrodes were secured to the right atrial appendage and connected to a pacemaker (model 5320; Medtronic, Inc, Minneapolis, Minn). Heart rate was maintained at a constant rate of 200 beats/min. Pressure and electrogram tracings were displayed continuously and digitized in real time on a computer by means of a waveform recording system (WinDAQ/400; DATAQ Instruments, Akron, Ohio) at a sampling rate of $2000 \mathrm{~Hz}$. A needle temperature probe (model BAT 8; Bailey Instruments, Saddle Brook, NJ) was placed in the right ventricle, which allowed myocardial temperature monitoring (model TM147T; Electromedics, Inc, Parker, Colo). The heart was enclosed in a plastic jacket to isolate from temperature deviations in the air and was maintained at $37^{\circ} \mathrm{C}$ by means of a water-jacketed beaker connected to a warm water bath (model D1/L; Haake Co, Berlin, Germany).

Experimental preparation. A crystalloid-perfused Langendorff apparatus was used for this study. The isolated rabbit heart was suspended by means of an aortic cannula at a column height of $70 \mathrm{~cm}$. The column was perfused with $37^{\circ} \mathrm{C}$ Krebs-Henseleit buffered solution, which consisted of the following: $\mathrm{NaCl}, 118.5 \mathrm{mmol} / \mathrm{L} ; \mathrm{NaHCO}_{3}, 25.0$ $\mathrm{mmol} / \mathrm{L} ; \mathrm{KCl}, 3.2 \mathrm{mmol} / \mathrm{L} ; \mathrm{KH}_{2} \mathrm{PO}_{4}, 1.2 \mathrm{mmol} / \mathrm{L} ; \mathrm{MgSO}_{4}$, $1.2 \mathrm{mmol} / \mathrm{L} ; \mathrm{CaCl}_{2}, 2.5 \mathrm{mmol} / \mathrm{L}$; and glucose, $5.5 \mathrm{mmol} / \mathrm{L}$. The Krebs-Henseleit solution was maintained at a strict $\mathrm{pH}$ range between 7.42 and 7.48 by bubbling with a gas mixture of $95 \%$ oxygen and $5 \%$ carbon dioxide. An ultrasonic flow probe (model T101; Transonic Systems Inc, Ithaca, NY) measured coronary flow in the perfusion column. A separate $70-\mathrm{cm}$ cardioplegic arrest column was maintained at $4^{\circ} \mathrm{C}$ by using water-cooling jackets. Water baths maintained the temperatures of the perfusion and cardioplegic arrest columns (models 800 and 9010; Fischer Scientific, Pittsburgh, Pa).

Experimental protocol. The isolated rabbit hearts underwent a 30-minute equilibration period after being mounted onto the Langendorff circuit. Baseline LV pressure-volume curves were then generated by inflating the LV balloon. Hearts were excluded from the study if they were unable to generate pressures of at least $60 / 10 \mathrm{~mm} \mathrm{Hg}$ during baseline data acquisition. Intracavitary LV pressures and bipolar LV electrograms were recorded over 7 balloon volumes (BVs), each corresponding to a fixed intracavitary LV end-diastolic pressure (EDP; 0, 2.5, 5, $10,15,20$, and $25 \mathrm{~mm} \mathrm{Hg}$ ). Crystalloid column flow corresponding to coronary flow was measured at each of these LV pressures. After baseline data acquisition, the LV balloon was deflated to generate an EDP of $2.5 \mathrm{~mm} \mathrm{Hg}$.
After baseline data acquisition, hearts were arrested with $50 \mathrm{~mL}$ of hypothermic solution followed by 4 hours of hypothermic storage in $50 \mathrm{~mL}$ of the same solution. Twenty-four hearts were randomized to receive 1 of 3 preservative solutions: (1) prototype solution containing $0.5 \mathrm{mmol} / \mathrm{L}$ pinacidil, (2) prototype solution without pinacidil as a control, and (3) UW solution $(n=8$ for each group). Hearts were stored for 4 hours at between $5^{\circ} \mathrm{C}$ and $10^{\circ} \mathrm{C}$.

Each heart was returned to the Langendorff circuit and reperfused for 60 minutes after storage. After reperfusion, LV pressure-volume measurements were generated by means of the same BVs used for baseline data acquisition. The percentage recovery of developed pressure (DP), diastolic pressure-volume relationships, perfusate flow, and myocardial edema was compared among treatment groups. After reperfusion data acquisition, a portion of the LV apex was excised, blotted, weighed (balance model TM 400; Mettler Instruments, Corp, Hightstown, NJ), and dried (Isotemp Oven model 625G; Fischer Scientific, Houston, Tex) until a constant dry weight was reached. Myocardial edema was expressed as the percentage of tissue water according to the following equation: percentage tissue water $=$ (wet weight dry weight)/wet weight $\times 100$.

Solution preparation. Each component of the prototype preservation solution (Table I) was added to a mixture of deionized water and $12 \%$ pentastarch solution (Du Pont Critical Care, Waukegan, Ill). Pinacidil (Leo Pharmaceutical Products, Ltd, Ballerup, Denmark) was added from a stock $0.1 \mathrm{~mol} / \mathrm{L}$ pinacidil dissolved in dimethyl sulfoxide. Procaine, $5 \mathrm{mmol} / \mathrm{L}$, was added to the pinacidil and control solutions designated as cardioplegic flush. This was performed by adding stock $1.0 \mathrm{~mol} / \mathrm{L}$ procaine $\mathrm{HCl}$ dissolved in dimethyl sulfoxide. The control solution contained a higher mannitol concentration $(70 \mathrm{mmol} / \mathrm{L})$ than the pinacidil solution $(25 \mathrm{mmol} / \mathrm{L})$ to equilibrate higher osmolarity from the addition of $0.5 \mathrm{mmol} / \mathrm{L}$ pinacidil in dimethyl sulfoxide. UW solution was obtained as ViaSpan from Du Pont Merck Pharmaceuticals (Wilmington, Del). UW solution was modified with the standard $0.016 \mathrm{~g} / \mathrm{L}$ dexamethasone, 200,000 $\mathrm{U} / \mathrm{L}$ penicillin, and $40 \mathrm{U} / \mathrm{L}$ insulin.

Data analysis. Digitized pressure waveforms were analyzed by means of commercial software programs (Spectrum 2.0; Triton Technology, Inc, San Diego, Calif; Microsoft Excel 97; Microsoft Corp, Redmond, Wash).

End-systolic pressure. The end-systolic pressure (ESP) of a beat was defined as the maximum point of the digitized pressure waveform. The average ESP was calculated by averaging 20 seconds of data. Average ESPs were obtained for each $\mathrm{BV}$ at baseline and after reperfusion (postischemic). The ESP versus $\mathrm{BV}$ data for baseline and postischemic data were fitted to the linear ESP-volume relationship below by a leastsquares linear regression algorithm as follows: $\mathrm{ESP}=\mathrm{E}_{\max }$ $(\mathrm{BV})+\mathrm{k}$.

End-diastolic pressure. The EDP of a beat was defined as the point at which the increase in slope of the pressure waveform exceeded a threshold of $0.5 \mathrm{~mm} \mathrm{Hg} / \mathrm{ms}$. Average EDPs were obtained for each BV at baseline and after reperfusion 
(postischemic). The EDP versus BV data were fitted to the linear EDP-volume relationship by using a least-squares linear regression algorithm as follows: $\mathrm{EDP}=\mathrm{m}(\mathrm{BV})+\mathrm{b}$. Mean baseline and postischemic slopes were compared for each group. The diastolic slope ratio was calculated by dividing the baseline slope by the postischemic slope.

$D P$. DP in the left ventricle was calculated as the difference between ESP and EDP. The DP versus BV values for baseline and postischemic data were fitted to a linear pressure-volume relationship by the following linear regression algorithm: DP $=\mathrm{ESP}-\mathrm{EDP}=\left(\mathrm{E}_{\max }[\mathrm{BV}]+\mathrm{k}\right)-(\mathrm{m}[\mathrm{BV}]+\mathrm{b})$.

Recovery of $\mathrm{DP}$. The recovery of $\mathrm{DP}$, expressed as a percentage, was calculated as the ratio of the postischemic DP to the baseline DP at the corresponding BV. The average percentage recovery of DP was calculated by a program developed in our laboratory by means of the trapezoidal rule..$^{5}$

Minimal diastolic negative $d P / d T$. The diastolic minimal negative $\mathrm{dP} / \mathrm{dT}\left(\mathrm{dd}_{\min }\right)$ of a beat was calculated as the minimal derivative of $L V$ pressure during diastolic relaxation. The mean $\mathrm{dd}_{\text {min }}$ was calculated by averaging 20 seconds of data. Average $\mathrm{dd}_{\text {min }}$ values were obtained for each BV at baseline and after reperfusion (postischemic). The $\mathrm{dd}_{\text {min }}$ versus BV data for baseline and postischemic data were fitted to secondorder polynomial regressions. Each regression was integrated to calculate the area under the curve (AUC), ranging from minimum to maximum matched BV. To express the diastolic contractile function over multiple LV volumes, we calculated the percentage recovery of minimal diastolic negative $\mathrm{dP} / \mathrm{dT}$ as a ratio of the postischemic AUC divided by the baseline AUC multiplied by 100 .

Statistical analysis. Statistical analysis was performed by means of the computer software SigmaStat (version 2.03; Jandel Corp, San Rafael, Calif) and Microsoft Excel 97 (Microsoft Corp). Results are expressed as means \pm SEM. A paired $t$ test was used to compare preischemic to postischemic end points within groups. A 1-way analysis of variance (ANOVA) was used to compare multiple means. If a statistically significant difference was detected in the ANOVA, individual comparisons between groups were made by a Student-Newman-Keuls post test.

\section{Results}

Perfusate flow. There were no differences in baseline coronary flow rates among the 3 groups. The mean flow rate for the preservative solutions during arrest was $19.0 \pm 1.0 \mathrm{~mL} / \mathrm{min}$ for hearts perfused with $\mathrm{UW}$ solution. This was significantly slower than flow rates for hearts perfused with pinacidil and control solutions, with mean flows of $28.7 \pm 3.1 \mathrm{~mL} / \mathrm{min}$ and $29.3 \pm 2.9$ $\mathrm{mL} / \mathrm{min}$, respectively. The flow rates of both prototype solutions were statistically equivalent. Postischemic coronary flows were significantly decreased compared with baseline for each group. Hearts exposed to pinacidil solution decreased from $43.6 \pm 1.4 \mathrm{~mL} / \mathrm{min}$ to $33.6 \pm 1.6 \mathrm{~mL} / \mathrm{min}$ after reperfusion $(P<.001)$. Hearts perfused with control solution decreased from $44.3 \pm$
Table I. Composition of preservative solutions

\begin{tabular}{|c|c|c|c|}
\hline Component & $\begin{array}{c}\text { Pinacidil } \\
\text { solution }\end{array}$ & $\begin{array}{l}\text { Control } \\
\text { solution }\end{array}$ & $\begin{array}{c}U W \\
\text { solution }\end{array}$ \\
\hline $\mathrm{Na}(\mathrm{mmol} / \mathrm{L})$ & 55 & 55 & 30 \\
\hline $\mathrm{K}(\mathrm{mmol} / \mathrm{L})$ & 5 & 5 & 120 \\
\hline $\mathrm{Cl}(\mathrm{mmol} / \mathrm{L})$ & 5 & 5 & - \\
\hline $\mathrm{Ca}(\mathrm{mmol} / \mathrm{L})$ & 0.1 & 0.1 & - \\
\hline $\mathrm{Mg}(\mathrm{mmol} / \mathrm{L})$ & 10 & 10 & 5 \\
\hline $\mathrm{H}_{2} \mathrm{PO}_{4}(\mathrm{mmol} / \mathrm{L})$ & 5 & 5 & 25 \\
\hline $\mathrm{SO}_{4}(\mathrm{mmol} / \mathrm{L})$ & 10 & 10 & 5 \\
\hline Pinacidil (mmol/L) & 0.5 & - & - \\
\hline Procaine (mmol/L) & 5 & 5 & - \\
\hline Histidine (mmol/L) & 100 & 100 & - \\
\hline Pentastarch (g/L) & 40 & 40 & 50 \\
\hline Insulin (IU/L) & 100 & 100 & 40 \\
\hline Glucose (mmol/L) & 10 & 10 & - \\
\hline Mannitol (mmol/L) & 25 & 70 & - \\
\hline Lactobionate $(\mathrm{mmol} / \mathrm{L})$ & 50 & 50 & 100 \\
\hline Adenosine (g/L) & - & - & 1.34 \\
\hline Glutathione (g/L) & - & - & 0.92 \\
\hline Allopurinol (g/L) & - & - & 0.136 \\
\hline Penicillin (U/L) & - & - & 200,000 \\
\hline Dexamethasone (g/L) & - & - & 0.016 \\
\hline Osmolality (mOsm/kg) & 400 & 365 & 315 \\
\hline $\mathrm{pH}$ & 7.65 & 7.65 & 7.40 \\
\hline
\end{tabular}

$3.1 \mathrm{~mL} / \mathrm{min}$ to $29.8 \pm 2.8 \mathrm{~mL} / \mathrm{min}(P<.001)$. Hearts stored in UW solution decreased from $45.0 \pm 4.5$ $\mathrm{mL} / \mathrm{min}$ to $32.5 \pm 4.7 \mathrm{~mL} / \mathrm{min}(P<.001)$. Although there was a trend that the greatest recovery of baseline coronary flow was found in hearts exposed to pinacidil $(77.8 \% \pm 3.0 \%)$ compared with those exposed to the control $(66.8 \% \pm 2.4 \%)$ and UW solutions $(70.9 \% \pm$ $4.0 \%)$, the differences were not statistically significantly different $(P=.07)$.

Postischemic systolic function. Hearts generated mean DPs of $86 \pm 4 \mathrm{~mm} \mathrm{Hg}$ at baseline. The percentage recovery of DP, calculated by the trapezoidal rule to accommodate multiple isovolumic pressure-volume points, was used to express systolic LV function. Hearts arrested and stored in pinacidil solution exhibited significantly better recovery of DP $(92.4 \% \pm 4.5 \%)$ than control solution-stored $(74.9 \% \pm 3.4 \%, P=.01)$ or UW solution-stored hearts $(66.7 \% \pm 5.1 \%, P=.001$; Fig 1).

Postischemic diastolic function. The recovery of diastolic negative $\mathrm{dP} / \mathrm{dT}$ (Fig 2) was best preserved in hearts exposed to pinacidil solution $(104.4 \% \pm 10.2 \%)$ compared with the control $(80.2 \% \pm 4.2 \%, P=.034)$ and UW solutions $(71.7 \% \pm 7.0 \%, P=.015)$. Postischemic diastolic slopes were higher than baseline for the UW $(P=.013)$ and control solutions $(P=.047$, Table II). Postischemic diastolic slopes were not statistically significantly different from baseline for hearts 


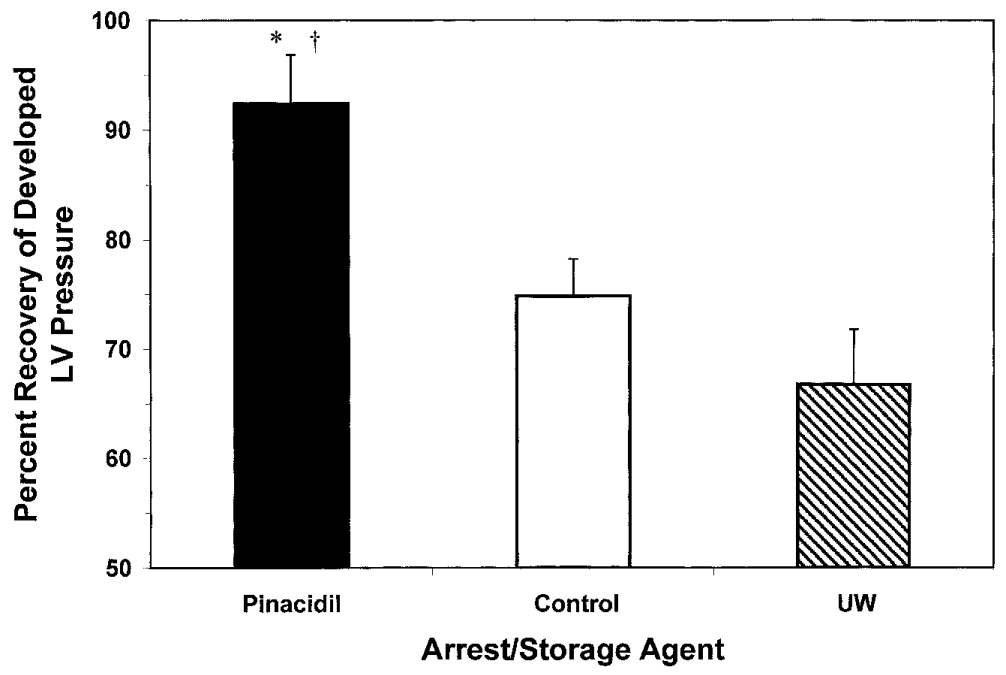

Fig 1. Postischemic systolic function expressed as the percentage of recovery of developed LV pressure of hearts arrested and stored for 4 hours in pinacidil solution, control solution, and UW solution. Values are means \pm SEM ( $\mathrm{n}=8$ for each group). There was a statistically significant difference determined by using 1-way ANOVA $(P=$ $.001)$ versus UW solution $(* P=.01)$ and versus control solution $(\dagger)$.

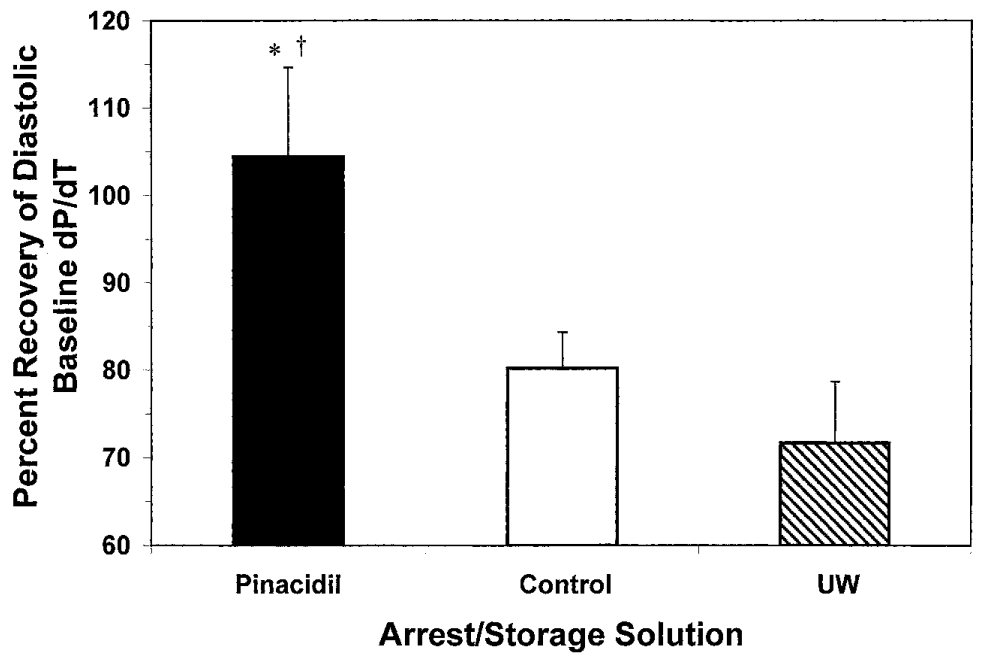

Fig 2. Postischemic diastolic function expressed as percentage of recovery of baseline diastolic negative dP/dT for hearts arrested and stored for 4 hours in pinacidil solution, control solution, and UW solution. Values are means \pm SEM ( $n=8$ for each group). Statistically significant differences determined by 1 -way ANOVA $(P=.015)$ versus UW solution $(* P=$ .034) and versus control solution $(\dagger)$.

exposed to the pinacidil preservation solution $(P=.093)$. Diastolic compliance, expressed as baseline/postischemic diastolic slope ratios (Fig 3), was worst preserved in hearts stored in UW solution $(0.67$ $\pm 0.07)$ compared with both the pinacidil $(0.88 \pm 0.05$, $P=.041)$ and control solutions $(0.87 \pm 0.05, P=.021)$.

Myocardial tissue water. There were no significant differences in percentage of tissue among the 3 experimental groups (Table II).

\section{Discussion}

Hyperpolarized arrest has emerged as an alternative approach to traditional depolarized arrest for prolonged storage of donor organs used in heart transplantation., Hyperpolarized arrest avoids many of the problems associated with hyperkalemic arrest by arresting the heart at its natural resting state, the resting membrane potential. Net intracellular ion fluxes are negligible at these potentials because transmembrane ion gradients are balanced, 


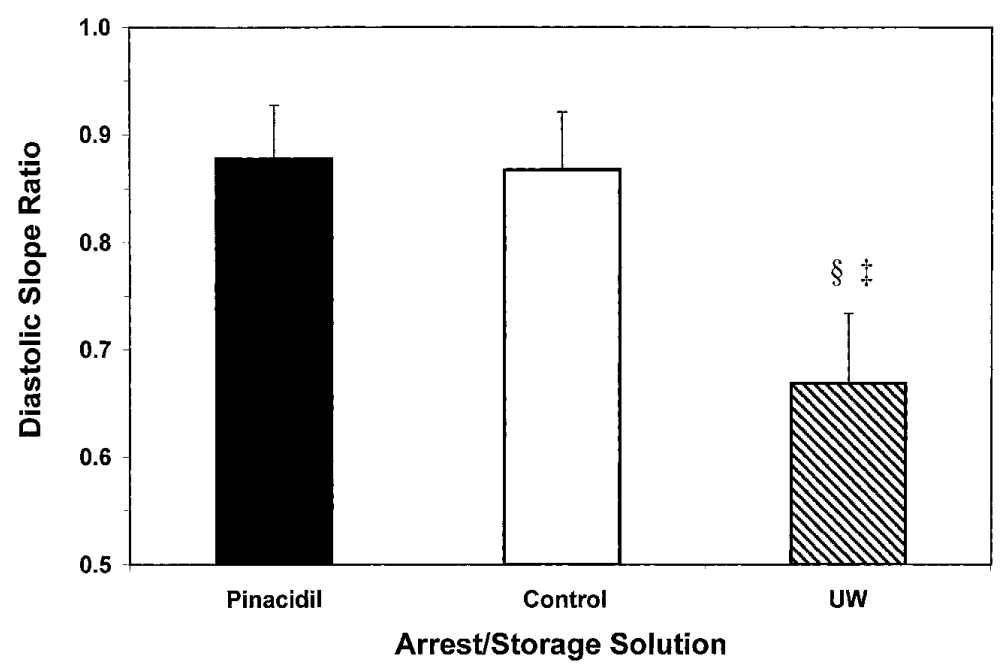

Fig 3. Postischemic diastolic compliance expressed as baseline/postischemic diastolic pressure-volume slope ratios for hearts arrested and stored for 4 hours in pinacidil solution, control solution, and UW solution. Values are means \pm SEM ( $n=8$ for each group). A ratio of 1 reflects no diastolic injury. Statistically significant differences determined by 1 -way ANOVA $(P=.041)$ versus pinacidil solution $(\S P=.021)$ and versus control solution $(\ddagger)$

Table II. Baseline and postischemic LV compliance and percentage tissue water

\begin{tabular}{lccc}
\hline Solution & Baseline diastolic slope & Postischemic diastolic slope & Percentage tissue water \\
\hline Pinacidil & $107.3 \pm 30.2$ & $120.7 \pm 33.2$ & $83.5 \pm 0.4$ \\
Control & $117.5 \pm 23.6$ & $137.8 \pm 28.2^{*}$ & $83.8 \pm 0.5$ \\
UW & $86.5 \pm 9.0$ & $138.2 \pm 20.5^{*}$ & $84.1 \pm 0.4$ \\
\hline
\end{tabular}

Values are means \pm SEM.

*Statistically different from baseline diastolic slope (paired $t$ test, $P=.047$ for control solution and $P=.013$ for UW solution).

and few voltage-gated ion channels are open. ${ }^{4}$ Accordingly, energy-dependent ion pump activity and metabolic demand are minimal. Studies have confirmed myocardial oxygen consumption during hyperpolarized arrest to be $50 \%$ less than depolarized arrest. ${ }^{8}$

Experimental evidence with several different pharmacologic agents has confirmed the benefits of hyperpolarized arrest. Snabaitis and associates ${ }^{9}$ demonstrated that hearts arrested with the sodium-channel blocker tetrodotoxin maintained resting membrane potential during hypothermic storage. These hearts exhibited better postischemic function than did hearts arrested and stored in hyperkalemic depolarizing solution. ${ }^{9}$ Hyperpolarized arrest facilitated by the opening of ATP-sensitive potassium channels by the cyanoguanidine pinacidil has also provided better cardioprotection than depolarized arrest. ${ }^{6,7}$ Hearts stored in pinacidil solution demonstrated improved postischemic function compared with hyperkalemic depolarizing solutions, such as St Thomas' Hospital solution, with cold ischemic times up to 2 hours. ${ }^{7}$ A recent study showed that these cardioprotective effects occur with longer 4-hour ischemic periods. ${ }^{5}$
The pharmacologic benefits of pinacidil stem from activation of an outward potassium that plays a role in the cells' normal response to ischemia. ${ }^{4}$ During ischemia, channels associated with this potassium current are triggered to open when intracellular levels of ATP decrease. Under nonischemic conditions, these channels are closed. Exogenous administration of pinacidil and other potassium-channel openers open these ATP-sensitive channels. This decreases membrane potential and the action potential duration, limiting calcium entry into the cytosol through voltage-sensitive calcium channels. ${ }^{4}$ Pinacidil also is a potent vasodilator that increases coronary flow in both nonischemic and ischemic hearts. ${ }^{5} \mathrm{~K}_{\mathrm{ATP}}$-mediated hyperpolarization of endothelial cells with subsequent smooth muscle relaxation has been shown to underly these vascular effects. ${ }^{16}$

Formulation of the pinacidil vehicle. Previous work in our laboratory has documented the efficacy of pinacidil administered in physiologic buffer (KrebsHenseleit) for long-term donor heart preservation. ${ }^{5}$ However, the clinical utility of these findings was limited because UW solution preserved postischemic con- 
tractile function better than pinacidil mixed in KrebsHenseleit solution. This prompted our formulation of a new hyperpolarizing solution that would optimize delivery of pinacidil and (1) reduce pathologic intracellular calcium accumulation, (2) prevent cell swelling, and (3) promote anaerobic glycolytic flux.

Intracellular calcium accumulation was hypothetically minimized during ischemia and reperfusion by decreasing the availability of extracellular calcium and reducing intracellular movement of extracellular calcium through voltage-sensitive calcium channels and exchangers. To limit availability of extracellular calcium, we formulated the solution with a low (100 $\mu \mathrm{mol} / \mathrm{L}$ ) concentration of calcium. This low concentration was chosen to prevent the calcium paradox associated with calcium-free solutions. ${ }^{17}$ Lactobionate was included because of its calcium-chelating activities. ${ }^{18}$ The lactobionate concentration was less $(50 \mathrm{mmol} / \mathrm{L})$ than that of UW solution (100 mmol/L); however, this lower concentration is equally efficacious in chelating calcium. ${ }^{18}$ The potassium concentration was maintained at normal serum levels $(5 \mathrm{mmol} / \mathrm{L})$ to prevent membrane depolarization, decreasing the ratio of open voltage-sensitive L-type calcium channels. Because high magnesium levels have been shown to displace calcium from sarcolemmal binding sites, a high magnesium level $(10 \mathrm{mmol} / \mathrm{L})$ was also included. ${ }^{19}$

Decreasing calcium entry during reperfusion by the sodium-calcium exchanger was an additional strategy in the formulation of the hyperpolarizing solution. Under nonischemic and nonhypothermic conditions, intracellular sodium is extruded through the sodiumpotassium adenosine triphosphatase. Hypothermia and ischemia render this pump virtually nonfunctional. ${ }^{4}$ Intracellular sodium levels rise during ischemia, ${ }^{20}$ which are effectively extruded by the bidirectional sodium-calcium exchanger during reperfusion. Efforts to prevent intracellular sodium accumulation in our solution focused on (1) decreasing the sodium concentration gradient by providing a low $(55 \mathrm{mmol} / \mathrm{L})$ sodium concentration, (2) preventing entry by blocking sodium channels with procaine, and (3) decreasing exchange of extracellular sodium for intracellular protons through the sodium-proton exchanger by robust buffering of intracellular $\mathrm{H}^{+}$. Because the sodium-proton exchanger is the major compensatory mechanism of myocytes to regulate increases in intracellular protons,${ }^{21}$ the intracellular proton-buffering effect of histidine was believed to be important. ${ }^{15}$

The activity of the $\mathrm{Na}^{+} / \mathrm{Ca}^{++}$antiporter during hypothermia is unknown. However, it is generally agreed that sodium-calcium exchange likely occurs mostly during reperfusion and rewarming. The precise activity of the sodium-proton exchanger during hypothermia also remains controversial. Although it has been demonstrated that specific blockade of this exchanger during ischemia results in improved postischemic LV function, sodium-proton exchange blockade during reperfusion provides more robust improvement of postischemic function..$^{22}$

Several components of this solution were included to protect against myocyte swelling. Because chloride has been shown to function as a primary trigger for myocyte swelling during hypothermic cardioplegic arrest, ${ }^{12,14}$ the chloride concentration was minimized, adding only a nominal concentration necessary for procaine $\mathrm{HCl}$ addition $(5 \mathrm{mmol} / \mathrm{L})$. Hydroxyethyl starch and lactobionate were added to increase extracellular osmotic bulk. ${ }^{13}$ Mannitol was included for its welldescribed free-radical scavenging properties ${ }^{23}$ in addition to increasing and balancing osmolarity.

Histidine also was added to promote anaerobic glycolytic flux. ${ }^{15}$ Ohkado and colleagues ${ }^{15}$ have shown that the intracellular buffering action of histidine increased the anaerobic end-products alanine and lactate, which correlated with improved postischemic function. This was facilitated when the metabolic substrate glucose was included in the preservation solution along with insulin to facilitate intracellular glucose entry. Inclusion of both proton-buffering agent and glucose in preservation solutions would promote anaerobic glycolysis and ATP production to continue during ischemia. ${ }^{24}$

Recovery of systolic function. The control solution effectively preserved postischemic systolic function and was comparable with UW solution. The efficacy of the control solution could be related to the aforementioned principles of its formulation. However, the precise contribution of each of the components is not known. Furthermore, precise comparisons of the mechanism of action between these two solutions is complex because of the numerous formulation differences.

The most important finding of this study was that the addition of the potassium-channel opener pinacidil to the control solution resulted in significantly improved postischemic systolic function of hearts when compared with UW solution. This finding supports our hypothesis that hyperpolarized arrest through opening of $\mathrm{K}_{\text {ATP }}$ channels may offer superior preservation of postischemic contractile function when compared with traditional hyperkalemic depolarized arrest. This supports previous findings examining hyperpolarized cardioplegic arrest during shorter periods of ischemia. ${ }^{6,12}$ It is not clear whether the protective effect of pinacidil is mediated through the sarcolemmal or mitochondrial $\mathrm{K}_{\mathrm{ATP}}$ channels. Recent stud- 
ies have documented that activation of mitochondrial $\mathrm{K}_{\text {ATP }}$ channels is cardioprotective. ${ }^{25}$ In this study we were unable to discern which $\mathrm{K}_{\text {ATP }}$ channel is more important because pinacidil nonselectively activates channels on both sarcolemma and mitochondria. ${ }^{25}$

Recovery of diastolic function. The control solution also effectively preserved postischemic diastolic function equivalent to UW solution. However, with the addition of pinacidil, the hyperpolarizing solution was able to significantly surpass the performance of hearts stored in either UW solution or the control solution. This preservation of active relaxation was assessed by the percentage recovery of diastolic negative $\mathrm{dP} / \mathrm{dT}$ over several LV BVs. The negative $\mathrm{dP} / \mathrm{dT}$ is influenced by the rate at which calcium is removed from the contractile proteins by the sarcoplasmic reticulum calcium-adenosine triphosphatase pump. ${ }^{26}$ Although direct measurement is lacking, our results suggest more efficient handling of calcium in hearts exposed to pinacidil solution. This hypothesis is supported by the fact that sarcoplasmic reticulum calcium-adenosine triphosphatase pumps have been shown to be sensitized by other potassium-channel openers, such as nicorandil, which correlated with enhanced lusitropy. ${ }^{27}$

Diastolic compliance was also best preserved by the pinacidil solution. This was assessed by comparing diastolic slope ratios over several LV BVs. Contrary to preservation of active relaxation, optimized preservation of diastolic compliance was not solely attributable to pinacidil. Both the pinacidil solution and the pinacidil-free control solution preserved diastolic compliance significantly better than UW solution. This may have been attributable to their higher osmolarity. Although all 3 solutions shared the large molecular weight hydroxyethyl starch and lactobionate components, both prototype solutions were fashioned with a third impermeant, mannitol. This effectively raised the osmolarity of pinacidil and control solutions higher than UW solution ( $400 \mathrm{mOsm} / \mathrm{kg}$ and $365 \mathrm{mOsm} / \mathrm{kg}$ vs $315 \mathrm{mOsm} / \mathrm{kg}$, respectively). The benefits of increased osmolarity center around prevention of cellular swelling and preservation of the extracellular vascular space. Similar to our results, Starr and associates ${ }^{28}$ showed that diastolic compliance was a function of cardioplegic solution osmolarity. Myocardial tissue water was inversely related to compliance in that study. The finding that differences in the percentage of tissue water were not revealed in this study may relate to experimental protocol differences. ${ }^{28}$

Coronary flow. Hearts exposed to the pinacidil solution displayed improved postischemic coronary flow compared with control solution and UW solution, although these differences could be due to chance ( $P=$ .07). Pinacidil has been shown to protect against the endothelium-dependent vasodilatation impairment produced by ischemia through a $\mathrm{K}_{\mathrm{ATP}}$-dependent mechanism. ${ }^{29}$ The protective effect of other potassium-channel openers has been recently related to preservation of endothelium-derived hyperpolarizing factor-mediated coronary vasorelaxation. ${ }^{16}$ The vasodilatory properties of pinacidil produced by hyperpolarization of endothelial cells and subsequent vascular smooth muscle relaxation also provide a means for facilitation of myocardial perfusion during the reperfusion period. A relationship between improved systolic function and coronary flow has been previously documented by our group. ${ }^{5}$ However, the current study was unable to significantly correlate the improved systolic function and coronary flow with pinacidil solution. This may represent a type II statistical error because of the small sample size. Further studies will be needed to clarify this issue.

Limitations of the model. The crystalloid-perfused Langendorff preparation was used in this study because it allowed for analysis of interventions of the isolated heart without humoral, neural, adrenergic, or anesthetic influences. The crystalloid preparation is less complicated than a blood-perfused parabiotic animal preparation because of its independence from the physiologic variations produced by the support animal. Moreover, for long-term preservation of hearts, the maintenance of the support animal becomes logistically impossible. The findings of this study should be interpreted with caution because the current preparation lacked blood perfusion and the important dynamics of the intact organism. As with any experimental results, these findings should be extrapolated with caution to the clinical scenario.

An additional limitation of the study is based on the lack of direct measurements of intracellular calcium accumulation during ischemia and reperfusion. With the novel pharmacologic milieu produced by the hyperpolarizing solution during hypothermic storage, the precise contribution of each component of the pinacidil vehicle is unknown. An isolated comparison of each of the individual components that differed between the hyperpolarizing pinacidil and UW solutions was not possible. However, UW solution does provide a clinical frame of reference for comparison in these studies. It is considered the gold standard for donor heart preservation. These limitations will serve as the focus for future studies.

\section{Summary}

A novel solution was formulated to preserve donor hearts by using hyperpolarized arrest with the potassi- 
um-channel opener pinacidil. The delivery vehicle was carefully composed to prevent intracellular calcium accumulation and myocyte swelling and also promote anaerobic glycolytic flux. This investigation demonstrated $\mathrm{K}_{\text {ATP }}$ channel-mediated enhancement of postischemic systolic function, active diastolic properties, and coronary flow by addition of pinacidil to the control solution. Compared with UW solution, the pinacidil hyperpolarizing solution resulted in significantly better myocardial preservation of both systolic function and the diastolic properties of the left ventricle. This study documents that potassium-channel openers are effective for donor heart preservation and have advantages over UW solution. Future studies are needed to clarify whether these solutions may prolong the period of safe myocardial protection in cardiac transplantation.

\section{REFERENCES}

1. Darracott-Cankovic S, Stovin PG, Wheeldon D, Wallwork J, Wells F, English TA. Effect of donor heart damage on survival after transplantation. Eur J Cardiothorac Surg 1989;3:525-32.

2. Duquesnoy RJ, Cramer DV. Immunologic mechanisms of cardiac rejection. Cardiovasc Clin 1990;20:87-103.

3. Gettes LS, Cascia WE. Effect of acute ischemia on cardiac electrophysiology. In: Fozzard HA, Haber E, Jennings RB, Katz AM, Morgan HE, editors. The heart and cardiovascular system. New York: Raven; 1992. p. 2021-54.

4. Damiano RJ. The electrophysiology of ischemia and cardioplegia: implications for myocardial protection. J Card Surg 1995;10(Suppl):101-9.

5. Hoenicke EM, Peterseim DS, Ducko CT, Sun X, Damiano RJ Jr. Donor heart preservation with the potassium channel opener pinacidil: comparison to University of Wisconsin and St. Thomas' solution. J Heart Lung Transplant 2000;19:286-97.

6. Jayawant M, Stephenson ER Jr, Damiano RJ Jr. Advantages of continuous hyperpolarized arrest with pinacidil over St. Thomas' Hospital solution during prolonged ischemia. J Thorac Cardiovasc Surg 1998;116:131-8.

7. Jayawant AM, Stephenson ER Jr, Matte GS, Prophet GA, LaNoue KF, Griffith JW, et al. Potassium-channel opener cardioplegia is superior to St. Thomas' solution in the intact animal. Ann Thorac Surg 1999;68:67-74.

8. Sternbergh WC, Brunsting LA, Abd-Elfattah AS, Wechsler AS. Basal metabolic energy requirements of polarized and depolarized arrest in rat heart. Am J Physiol 1989;265:H846-51.

9. Snabaitis AK, Shattock MJ, Chamber DJ. Comparison of polarized and depolarized arrest in the isolated rat heart for long-term preservation. Circulation 1997;96:3148-56.

10. Sun X, Ducko CT, Hoenicke EM, Zhang X-Q, Cheung JY, Damiano RJ Jr. Potassium channel openers avoid cardioplegiainduced intracellular calcium accumulation but do not prevent myocardial stunning. Circulation 1999;100(Suppl):I-388.

11. Matsuda N, Tofukuji M, Morgan KG, Sellke FW. Coronary microvascular protection with $\mathrm{Mg}^{2+}$ : effects on intracellular calcium regulation and vascular function. Am $\mathrm{J}$ Physiol 1999;276:H1124-30.

12. Jayawant AM, Stephenson ER, Baumgarten CM, Damiano RJ Jr. Prevention of cell swelling with low chloride St. Thomas'
Hospital solution improves postischemic myocardial recovery. J Thorac Cardiovasc Surg 1998;115:1196-202.

13. Southard JH, Belzer FO. Organ preservation. Annu Rev Med 1995;46:235-47.

14. Hoenicke EM, Sun X, Ducko CT, Damiano RJ Jr. Prevention of myocyte edema with University of Wisconsin Solution: a potential mechanism for improved donor heart preservation. Surg Forum 1999;50:181-2.

15. Ohkado A, Hung C-D, Sommers E, del Nido PJ. Evaluation of highly buffered low-calcium solution for long-term preservation of the heart: comparison with University of Wisconsin solution. J Thorac Cardiovasc Surg 1994;108:762-71.

16. He G-W. Potassium-channel opener in cardioplegia may restore coronary endothelial function. Ann Thorac Surg 1998;66:131822.

17. Fremes SE, Zhang J, Furukawa RD, Mickle DA, Weisel RD. Cardiac storage with University of Wisconsin solution, calcium, and magnesium. J Heart Lung Transplant 1995;14:916-25.

18. Burgmann H, Reckendorfer H, Sperlich M, Doleschel W, Spieckermann PG. The calcium chelating capacity of different protecting solutions. Transplantation 1992;54:1106-8.

19. Lansman JB, Hess P, Tsien RW. Blockade of current through single calcium channels by $\mathrm{Ca}^{2+}, \mathrm{Mg}^{2+}$, and $\mathrm{Cd}^{2+}$ voltage and concentration dependence of calcium entry into the pore. J Gen Physiol 1986;88:321-47.

20. Schepkin VD, Choy IO, Budinger TF, Young JN, DeCampli WM. Multi-dose crystalloid cardioplegia preserves intracellular sodium homeostasis in myocardium. J Mol Cell Cardiol 1999;31:1643-51.

21. Grace AA, Kirschenlohr HL, Metcalfe JC, Smith GA, Weissberg $\mathrm{PL}$, Cragoe EJ Jr, et al. Regulation of intracellular $\mathrm{pH}$ in the perfused heart by external $\mathrm{HCO} 3-$ and $\mathrm{Na}(+)-\mathrm{H}+$ exchange. Am J Physiol 1993;265:H289-98.

22. Myers ML, Karmazyn M. Improved cardiac function after prolonged hypothermic ischemia with the $\mathrm{Na}^{+} / \mathrm{H}^{+}$exchange inhibitor HOE 694. Ann Thorac Surg 1996;61:1400-6.

23. Ferreira R, Burgos M, Llesuy S, Molteni L, Milei J, Flecha BG, et al. Reduction of reperfusion injury with mannitol cardioplegia. Ann Thorac Surg 1989;48:77-84.

24. Takeuchi K, Hung C-D, Kawai A, Ohkado A, Konishi H, McGowan FX, et al. Prolonged preservation of the blood-perfused canine heart with glycolysis-promoting solution. Ann Thorac Surg 1999;68:903-7.

25. Gross GJ, Fryer RM. Sarcolemmal versus mitochondrial ATPsensitive $\mathrm{K}^{+}$channels and myocardial preconditioning. Circ Res 1999;84:973-9.

26. Katz AM. Indices of contractility and relaxation. In: Katz AM, ed. Physiology of the heart. New York: Raven Press; 1992. p. 397-414.

27. Irie H. Experimental studies on ischemic injury and reperfusion injury to the cardiac sarcoplasmic reticulum-the myocardial protective effect of nicorandil. Jpn Circ J 1988;52:563-9.

28. Starr JP, Jia C-X, Amirhamzeh MMR, Rabkin DG, Hart JP, Hsu DT, et al. Coronary perfusate composition influences diastolic properties, myocardial water content, and histologic characteristics of the rat left ventricle. Ann Thorac Surg 1999;68:925-30.

29. Maczewski M, Beresewicz A. The role of adenosine and ATPsensitive potassium channels in the protection afforded by ischemic preconditioning against the post-ischemic endothelial dysfunction in guinea-pig hearts. J Mol Cell Cardiol 1998;30:1735-47. 